\title{
REVIEWS \\ Community Health Worker Interventions to Improve Glycemic Control in People with Diabetes: A Systematic Review and Meta-Analysis
}

\author{
Walter Palmas, MD, MS, FAHA' , Dana March, $P h D^{2}$, Salima Darakjy, $M P H^{2}$, Sally E. Findley, $P h D^{3}$, \\ Jeanne Teresi, EdD, $P h D^{4,5}$, Olveen Carrasquillo, $M D^{6}$, and José A. Luchsinger, $M D^{1,2}$

\begin{abstract}
'Department of Medicine, Columbia University Medical Center, New York, NY, USA; ${ }^{2}$ Department of Epidemiology, Columbia University Mailman School of Public Health, New York, NY, USA; ${ }^{3}$ Department of Sociomedical Sciences, Columbia University Mailman School of Public Health, New York, NY, USA; ${ }^{4}$ New York State Psychiatric Institute, Columbia University Stroud Center, New York, NY, USA; ${ }^{5}$ Research Division, Hebrew Home at Riverdale, Bronx, NY, USA; ${ }^{\circ}$ Department of Medicine, University of Miami, Miami, FL, USA.
\end{abstract}

\begin{abstract}
OBJECTIVES: We set out to review the efficacy of Community Health Worker (CHW) interventions to improve glycemia in people with diabetes.

METHODS: Data sources included the Cochrane Central Register of Controlled Trials, Medline, clinicaltrials.gov, Google Scholar, and reference lists of previous publications. We reviewed randomized controlled trials (RCTs) that assessed the efficacy of CHW interventions, as compared to usual care, to lower hemoglobin Alc (A1c). Two investigators independently reviewed the RCTs and assessed their quality. Only RCTs with a follow-up of at least 12 months were meta-analyzed. A random effects model was used to estimate, from unadjusted withingroup mean reductions, the standardized mean difference (SMD) in Alc achieved by the CHW intervention, beyond usual care.
\end{abstract}

RESULTS: Thirteen RCTs were included in the narrative review, and nine of them, which had at least 12 months of follow-up, were included in the meta-analysis. Publication bias could not be ruled-out due to the small number of trials. Outcome heterogeneity was moderate $\left(\mathrm{I}^{2}=37 \%\right)$. The SMD in Alc (95\% confidence interval) was 0.21 (0.110.32). Meta-regression showed an association between higher baseline Alc and a larger effect size.

CONCLUSIONS: CHW interventions showed a modest reduction in Alc compared to usual care. Alc reduction was larger in studies with higher mean baseline Alc. Caution is warranted, given the small number of studies.

J Gen Intern Med 30(7):1004-12

DOI: $10.1007 / \mathrm{s} 11606-015-3247-0$

(c) Society of General Internal Medicine 2015

\section{INTRODUCTION}

Medically underserved populations, including racial minorities and/or people at the lower socioeconomic strata, are at higher risk of having diabetes mellitus, and tend to suffer complications from it more frequently. ${ }^{1,2}$ There is great

Received June 30, 2014

Revised February 2, 2015

Accepted February 6, 2015

Published online March 4, 2015 interest in developing and validating culturally sensitive approaches to improve diabetes care in underserved populations. Of particular interest are interventions to improve glycemic control, which is most often measured as a reduction in serum hemoglobin A1c levels (A1c, which reflects blood glucose levels over previous weeks).

Community health workers (CHWs, known in Spanish as Promotoras or Promotores de Salud) have been shown to improve health care delivery in several settings, including Latin America and the United States. ${ }^{3}$ CHWs have been utilized in health care delivery for underserved populations for decades because of their positive impact in multiple areas, including culturally appropriate delivery of health education, selfempowerment, and improved navigation of the health care system. CHWs have been shown to provide efficacious interventions in a wide range of health issues. A systematic review by Lewin et al. showed benefits for the promotion of breastfeeding and immunizations in children and adults. ${ }^{4}$ There was also evidence of reduced mortality in malaria and acute respiratory infections in underserved children. ${ }^{4}$ In diabetes, a review by Norris et al. reported improvements in diabetesrelated knowledge, self-care, and lifestyle. ${ }^{5}$ In regards to costeffectiveness, a study suggested that a CHW program in diabetes care could result in annual cost savings of around \$2,000 per Medicaid participant. ${ }^{6}$ Another study found that an outreach program for underserved men in Denver, $\mathrm{CO}$ could yield a return on investment of $\$ 2,28$ per dollar invested, through an optimization in outpatient care resource utilization. ${ }^{7}$

However, the value of CHW interventions to improve glycemic control in medically underserved populations remains unclear. Randomized controlled trials have had conflicting results. Some trials showed a significant reduction in A1c, ${ }^{8-}$ ${ }^{12}$ while other trials did not. ${ }^{13,14}$ Of note, there was methodological variability across studies, including sample size, target population, intervention protocols, and length of follow-up.

We conducted a systematic review of published randomized trials that assessed $\mathrm{CHW}$ interventions to improve diabetes care in underserved populations, including U.S. Hispanics, and provided a meta-analytic estimate of the A1c reduction achieved by those interventions, as compared to usual care, pooling data from studies with at least 12 months of follow-up. 


\section{METHODS}

We systematically reviewed published randomized controlled trials that assessed the efficacy of CHW interventions to lower A1c in underserved, at-risk (minority, and/or lower income) populations. We included all randomized controlled trials, published in English, of CHW interventions delivered in the outpatient care setting, alone or as part of a multidisciplinary care model, in adults (age 18 years or older) belonging to ethnic/racial minorities, and/or to a low socioeconomic stratum. The decision to include studies performed in different ethnic/racial groups was made due to the paucity of clinical trials in this field. The primary literature search was performed in MEDLINE, as described in Figure 1. We also performed additional searches in the Cochrane Central Registry of Controlled Trials, clinicaltrials.gov, Google Scholar, and examined the reference lists of pertinent studies. Those additional searches did not add any completed randomized controlled trial to those identified through the MEDLINE search. Our query included all data available up to 23 September 2104. We searched for the terms "community health workers", or "promotores", or promotoras", or "lay health workers", or "peer educators", or "peer education", or "health educators". We subsequently applied the PubMed filter "Clinical Trial", and selected the subgroup that included any of the following terms in the title, abstract, or the text of the publication: "diabetes", or "diabetic", or "glucose", or "glycemia", or "glycemic", or "blood sugar". This query identified 170 studies, for which abstracts or full manuscripts (as available) were reviewed by two investigators, and 157 were excluded because they had a non-randomized design. Full-text articles were available for all 13 of the identified randomized controlled trials. Only studies with at least 12 months of intervention and followup were included in the meta-analytic effect size estimate, although studies of shorter duration were included in the narrative review. When data on within-group mean reduction in A1c was not available in the original publication, it was kindly provided by the study investigators, upon our request.

The quality of individual trials, and risk of bias, were assessed independently by two of three investigators (W.P., D.M., or S.D.) at the study level, using the SIGN review checklist, and discrepancies were adjudicated by consensus. ${ }^{15}$ Funnel plots, which show the relationship between effect size and precision of the estimate, and Egger's regression test were plotted, but the number of studies was too small to properly assess for publication bias (the bias incurs when smaller negative trials are not published, but smaller positive trials are published). Heterogeneity of treatment outcomes (i.e., A1c reduction) across studies was assessed with the $\mathrm{I}^{2}$ statistic, which may be interpreted as representing low, moderate, or substantial heterogeneity for values of 0-25\%, 25-50\%, and 50-75\%, respectively. ${ }^{16}$ Treatment effects on glycemic control may vary depending on the population A1c levels at baseline, and greater effects may be seen in populations with higher A1c at randomization. ${ }^{17}$ Therefore, associations of treatment effect size with baseline A1c levels were assessed through metaregression. A conservative random effects model was used to pool the standardized mean reduction in A1c achieved by the CHW intervention, as compared to usual care. Hedges' adjusted $g$ was used to provide a common metric of treatment effect size regardless of the laboratory method used to measure A1c in each study, and to account for the relatively small sample sizes. The pooled effect size is thus summarized as the standardized mean difference between treatment arms, which measures the incremental A1c reduction by the intervention, above and beyond usual care, in standard deviation units. Significance of the pooled effect size estimate was tested with a $\mathrm{Z}$ test. Pooled estimates were obtained using the RevMan software, version 5.1 (Cochrane collaboration, Nordic Cochrane Center). ${ }^{18}$

\section{RESULTS}

The results of our literature search are described in Figure 1. We identified 13 completed randomized clinical trials. ${ }^{8-14,19-}$

${ }^{24}$ Table 1 describes the basic characteristics of those thirteen trials, including the setting for participant recruitment, duration of the study, and ethnicity of the study populations. Most of them studied minority populations, either AfricanAmerican, ${ }^{13}$ Hispanics, ${ }^{9,10,12,14,19,21,23,24}$ or both. ${ }^{8}$ Allen et al. recruited some White participants ( $21 \%$ of their study sample), but the majority of their participants were AfricanAmerican. ${ }^{11}$ DePue et al. recruited American Samoans. ${ }^{20}$

The components of the control arm varied substantially across studies. In two of the studies, participants in the control arm were placed on a waiting list, and received no additional care during the study. ${ }^{8,10}$ Lujan et al. assigned the control arm participants to receive their usual care at the clinic, ${ }^{9}$ whereas in the study by Corkery et al., the control arm participants received care from a certified diabetes educator. ${ }^{19}$ The study by Babamoto et al. had two control arms: in one control arm, the participants were managed by a diabetes nurse, and in the other control arm, participants continued to receive usual care from their primary care providers. ${ }^{14}$ In three of the longer studies, the usual care provided by primary care providers was "enhanced" by either feedback to participants and $\mathrm{PCP},{ }^{11}$ or regular telephone and mail contact with participants, with $^{13}$ or without ${ }^{21}$ feedback to the PCP. In the two-year-long MATCH study, participants in the control arm were mailed 36 monthly issues of a diabetes education newsletter. ${ }^{12,14}$ Finally, the design of the study by Tang et al. merits particular attention, because it deviates from the approach of comparing the CHW intervention to usual care. ${ }^{24}$ In that study, all participants first received a 6-month diabetes self-management education program. After that, participants were randomized to: 1) 12 months of weekly group sessions delivered by Peer Leaders, with telephone outreach to those unable to attend; or 2) 12 months of monthly telephone outreach by CHWs. It is thus apparent that the Peer Leader arm, which we consider as the "control arm" for the purposes of our review, was more contact intensive than the CHW arm, thus increasing the 


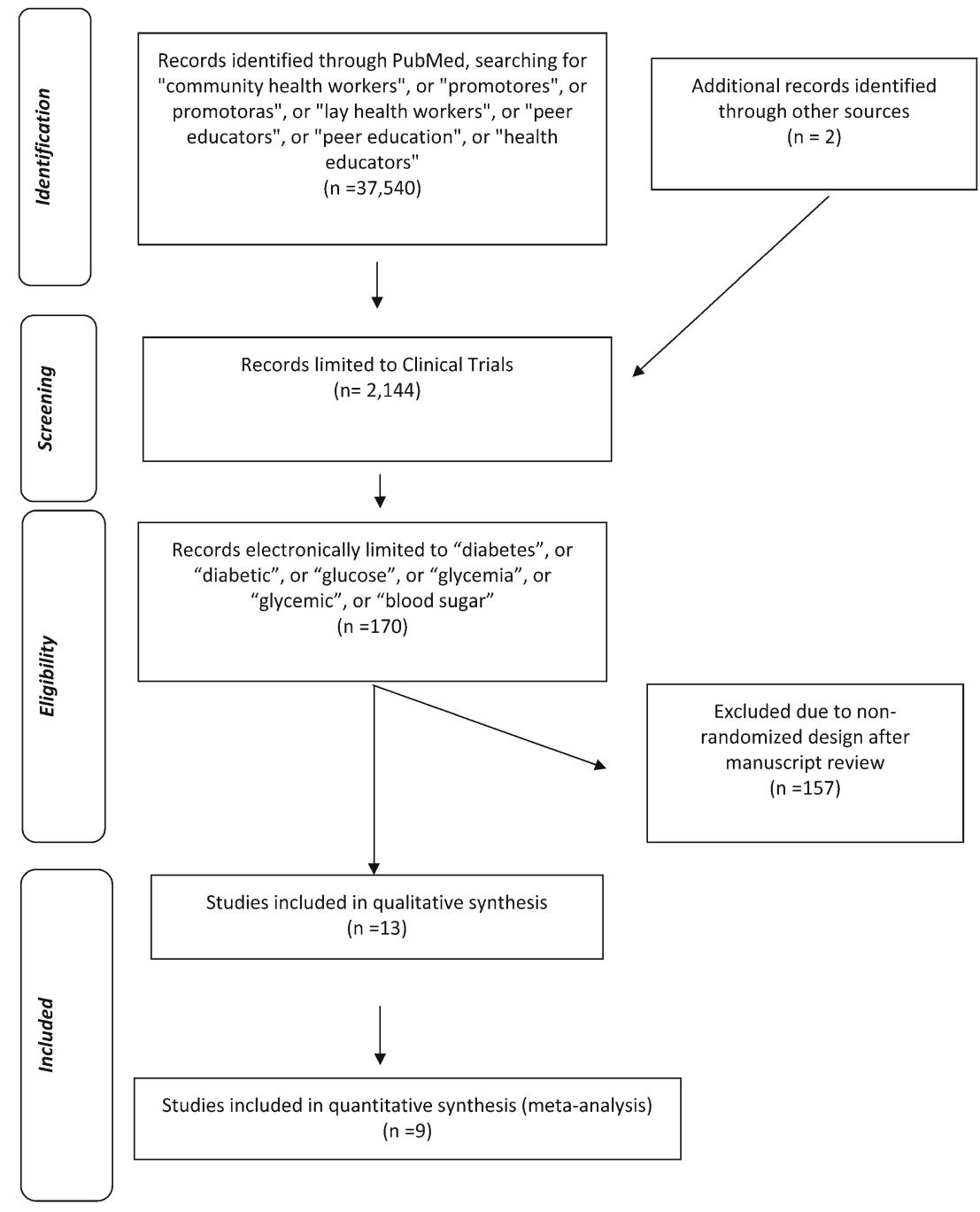

Figure 1. Literature search.

probability of showing greater relative efficacy for Peer Leaders. $^{24}$

The components of the CHW intervention also differed substantially from study to study. In eight of the studies, the CHWs worked on their own, whereas in the remaining cases, the CHW worked with either a certified diabetes educator, ${ }^{19}$ a nurse and a dietician, ${ }^{10}$ a nurse practitioner, ${ }^{11}$ or a nurse case manager. ${ }^{13,20}$ As described in Table 2, in all the studies, CHWs worked for a local non-profit organization. In most cases, they had received formal training in preparation for the study, and followed a well-defined intervention protocol. In nine of the trials, the CHW intervention protocol actively integrated the participant's Primary Care Provider. Seven out of 13 studies included group classes as part of the protocol. Telephone contacts were implemented in eight of the trials. The number of contacts warranted by the intervention protocol, either in person or by phone, was highly variable across studies, and the exact number of CHW-participant encounters that were actually carried out was not reported in most studies. Finally, only one study reported community outreach activities as a component of the intervention. ${ }^{12}$
With regard to the training CHWs received, the approach taken in certain studies is noteworthy, as it may provide a template for clinical implementation. For example, in the study by Prezio et al., the CHW was professionally certified by the state of Texas, and received 27 hours of additional instruction from Certified Diabetes Educators, Registered Dietitians, and an endocrinologist. ${ }^{23}$ She was also required to pass a written examination and a clinical examination supervised by the community diabetes instructors. In the MATCH (Mexican American Trial of Community Health Workers) study, the CHWs underwent stepwise training in Spanish. ${ }^{12}$ The initial phase consisted of a brief review of CHW practice, formal training on diabetes knowledge, and project-specific training on self-management and home visiting. The diabetes knowledge component required 24 hours of face-to-face training, and culminated in a written test assessing knowledge of diabetes self-management. CHWs were taught to use a home glucose monitor, interpret results, and understand the relationship between results and actions required based on results. Subsequent training was delivered by the MATCH research team, which consisted of 
Table 1. Randomized Controlled Trials of Community Health Worker Interventions for Diabetes Care

\begin{tabular}{|c|c|c|c|c|c|c|}
\hline First Author & Year & $\begin{array}{l}\text { Recruitment } \\
\text { Setting }\end{array}$ & $\begin{array}{l}\text { Population } \\
\text { Ethnicity }\end{array}$ & $\begin{array}{l}\text { Duration } \\
\text { (months) }\end{array}$ & $\begin{array}{l}\text { Control Arm } \\
\text { N = Sample Size; } \\
\text { Mean Baseline A1c }\end{array}$ & $\begin{array}{l}\text { Intervention } \\
\text { N = Sample Size; } \\
\text { Mean Baseline A1c }\end{array}$ \\
\hline Corkery $^{19}$ & 1997 & $\begin{array}{l}\text { Diabetes management } \\
\text { clinic at tertiary care } \\
\text { hospital; New York, } \\
\text { NY }\end{array}$ & Hispanics & $<6^{*}$ & $\begin{array}{l}\text { Certified Diabetes } \\
\text { Educator; } \mathrm{N}=34 ; \\
\text { Mean baseline A1c } \\
\text { not available. }\end{array}$ & $\begin{array}{l}\text { CHW with Certified } \\
\text { Diabetes Educator; } \\
\mathrm{N}=30 \text {; Mean baseline } \\
\text { A1c not available. }\end{array}$ \\
\hline Lujan $^{9}$ & 2007 & $\begin{array}{l}\text { Community clinic; } \\
\text { Southern Texas, TX }\end{array}$ & Hispanics & 6 & $\begin{array}{l}\text { Usual care at clinic; } \\
\mathrm{N}=74 ; 7.7 \%\end{array}$ & $\mathrm{CHW} ; \mathrm{N}=75 ; 8.7 \%$ \\
\hline Babamoto $^{14}$ & 2009 & $\begin{array}{l}\text { Three inner-city family } \\
\text { health centers; } \\
\text { Los Angeles, CA }\end{array}$ & Hispanics & 6 & $\begin{array}{l}\text { Two control arms: a) } \\
\text { Diabetes Nurse, b) PCP; } \\
\text { a) } \mathrm{N}=60 ; 8.5 \% \text {; b) } \\
\mathrm{N}=54 ; 9.5 \%\end{array}$ & $\begin{array}{l}\mathrm{CHW} \\
\mathrm{N}=75 ; 8.6 \%\end{array}$ \\
\hline Spencer $^{8}$ & 2011 & $\begin{array}{l}\text { Two community } \\
\text { health systems; } \\
\text { Detroit, MI }\end{array}$ & $\begin{array}{l}57 \% \text { African-American } \\
43 \% \text { Hispanics }\end{array}$ & 6 & $\begin{array}{l}\text { Delayed treatment } \\
\text { (waiting list); } \\
\mathrm{N}=92 ; 8.5 \%\end{array}$ & $\mathrm{CHW} ; \mathrm{N}=72 ; 8.6 \%$ \\
\hline Brown $^{10}$ & 2002 & $\begin{array}{l}\text { Sample of research } \\
\text { roster; Starr County, } \\
\text { TX }\end{array}$ & Hispanics & 12 & $\begin{array}{l}\text { Delayed treatment } \\
\text { (waiting list); } \\
\mathrm{N}=126 ; 11.8 \%\end{array}$ & $\begin{array}{l}\text { CHW with Nurse } \\
\text { and Dietician; } \\
\mathrm{N}=126 ; 11.8 \%\end{array}$ \\
\hline Gary $^{13}$ & 2009 & $\begin{array}{l}\text { University-affiliated } \\
\text { managed care; } \\
\text { Baltimore, MD }\end{array}$ & African-American & 24 & $\begin{array}{l}\text { Feedback to PCP (once); } \\
\text { mailings, calls to } \\
\text { subjects (every } 6 \text { months); } \\
\mathrm{N}=273 ; 8.0 \%\end{array}$ & $\begin{array}{l}\text { CHW with Nurse } \\
\text { Case Manager; } \\
\mathrm{N}=269 ; 7.7 \%\end{array}$ \\
\hline Allen $^{11}$ & 2011 & $\begin{array}{l}\text { Two community } \\
\text { health centers; } \\
\text { Baltimore, MD }\end{array}$ & $\begin{array}{l}79 \% \text { African-American, } \\
21 \% \text { White }\end{array}$ & 12 & $\begin{array}{l}\text { Feedback to participant } \\
\text { and PCP; } \mathrm{N}=264 ; 8.3 \%\end{array}$ & $\begin{array}{l}\text { CHW with Nurse } \\
\text { Practitioner; } \\
\mathrm{N}=261 ; 8.9 \%\end{array}$ \\
\hline Prezio $^{23}$ & 2013 & $\begin{array}{l}\text { Community clinic; } \\
\text { Dallas, TX }\end{array}$ & Hispanics & 12 & Usual care; $\mathrm{N}=90 ; 8.7 \%$ & $\mathrm{CHW} ; \mathrm{N}=90 ; 8.9 \%$ \\
\hline DePue $^{20}$ & 2013 & $\begin{array}{l}\text { Community health } \\
\text { center; American } \\
\text { Samoa, AS }\end{array}$ & Samoan & 12 & $\begin{array}{l}\text { Usual care (risk profile } \\
\text { assessment placed in } \\
\text { chart); } \mathrm{N}=164 ; 10.0 \%\end{array}$ & $\begin{array}{l}\text { CHW supervised } \\
\text { by Nurse Case } \\
\text { Manager; } \\
\mathrm{N}=104 ; 9.6 \%\end{array}$ \\
\hline Rothschild $^{12}$ & 2014 & $\begin{array}{l}\text { Metropolitan } \\
\text { Chicago, IL }\end{array}$ & Hispanics & 24 & $\begin{array}{l}\text { Monthly mailings; } \\
\mathrm{N}=71 ; 8.1 \%\end{array}$ & $\mathrm{CHW} ; \mathrm{N}=73 ; 8.3 \%$ \\
\hline Perez-Escamilla ${ }^{22}$ & 2014 & $\begin{array}{l}\text { Community clinic; } \\
\text { Hartford, CT }\end{array}$ & Hispanics & 12 & $\begin{array}{l}\text { Usual care; } \\
\mathrm{N}=106 ; 9.6 \%\end{array}$ & $\begin{array}{l}\text { CHW with Nurse, } \\
\text { Medical Assistant; } \\
\mathrm{N}=105 ; 9.6 \%\end{array}$ \\
\hline Tang $^{21}$ & 2014 & $\begin{array}{l}\text { Community health } \\
\text { center; Detroit, MI }\end{array}$ & Hispanics & 12 & $\begin{array}{l}\text { Peer leaders; } \\
\mathrm{N}=60 ; 8.2 \%\end{array}$ & $\mathrm{CHW} ; \mathrm{N}=56 ; 7.8 \%$ \\
\hline Palmas $^{21}$ & 2014 & $\begin{array}{l}\text { University-affiliated } \\
\text { community clinic; } \\
\text { New York, NY }\end{array}$ & Hispanics & 12 & $\begin{array}{l}\text { Quarterly mailings, } \\
\text { phone calls; } \mathrm{N}=179 \text {; } \\
8.6 \%\end{array}$ & $\mathrm{CHW} ; \mathrm{N}=181 ; 8.8 \%$ \\
\hline
\end{tabular}

*Education in the control arm lasted for a mean (range) of 3.4 (0.9-5.4) months; CHW Community Health Worker; PCP Primary Care Provider

two physicians, a nurse, and a clinical psychologist. It focused on: 1) application of diabetes information, and 2) self-management skills. A training manual provided written materials to accompany the didactic sessions. CHWs were evaluated via post-tests for adequate level of knowledge, and an assessment of competence via role play that documented increased skills, as well as areas for further training.

The individual studies varied substantially in quality, and the risk of bias was substantial for several of them, as we summarize in Table 3. As expected, given the nature of the intervention, none of the trials were double-blinded. Only six of the trials reported using adequate allocation concealment during randomization. Participant attrition rates were high in some studies, reaching $50 \%$ in the usual care (control) group of the trial performed by Babamoto et al. ${ }^{14}$ Only nine trials had a clinically meaningful follow-up of at least 12 months. ${ }^{10-13,20-24}$ Of note, those nine studies also exhibited higher quality, and substantially lower risk of bias, than shorter-term studies. For example, all of them described the standardized method applied to measure A1c, and they all followed the intention to treat principle to analyze A1c changes. In addition, those longer-term studies tended to have larger samples that enhanced their statistical power, and either achieved a balanced distribution of baseline characteristics across the randomized groups, or adjusted appropriately for them in the analysis. ${ }^{10-13,20-24}$

\section{META-ANALYSIS OF LONGER-TERM STUDIES}

We pooled the results of nine longer-term trials using a random effects model to compare unadjusted within-group mean A1c reduction in the CHW intervention arms to usual care. A funnel plot, and Egger's test, could not rule out publication bias, because of the small number of studies. ${ }^{25,26}$ Outcome heterogeneity was acceptable, with an $\mathrm{I}^{2}=37 \%$. The pooled standardized mean difference ( $95 \%$ confidence interval) achieved by CHW interventions, above and beyond usual care, was 0.21 $(0.11,0.32)$, as shown in Table 4 and Figure 2. In metaregression, there was an association between higher baseline A1c levels and a larger effect size, beta coefficient $(95 \%$ confidence interval $)=0.14(0.01,0,27)$. Because of lack of data, we were not able to assess whether a greater number of encounters between CHWs and participants resulted in greater A1c reduction. However, the two long-term studies with 


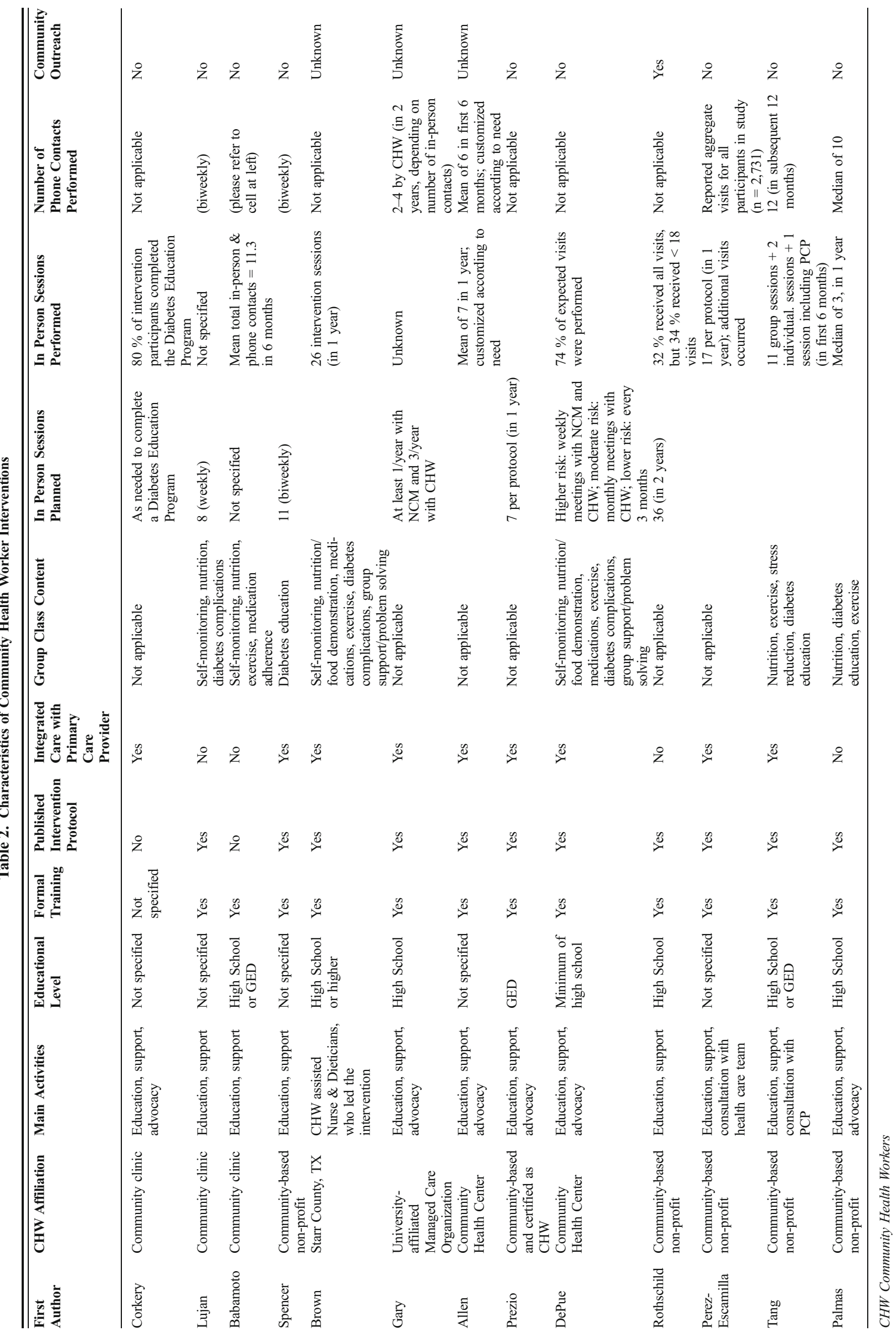


protocols requiring the largest number of CHW-participant visits, those by Brown et al. and Rotschild et al., ${ }^{10,12}$ did report the largest A1c reduction.

\section{DISCUSSION}

Our meta-analysis suggests that $\mathrm{CHW}$ interventions lasting at least 12 months result in a modest reduction in $\mathrm{A} 1 \mathrm{c}$, as compared to usual care. We found evidence in metaregression that greater A1c reduction may be achieved in populations with higher A1c levels at baseline. It is also possible that studies with a more visit-intensive $\mathrm{CHW}$ protocol might have shown greater efficacy. Unfortunately, detailed data describing the exact number of CHW-participant encounters was not available for several studies, and this precluded a meta-regression analysis to assess whether efficacy varied significantly according the intensity of the intervention.

Given that studies used different laboratory methods to measure A1c, we estimated the pooled effect size as standardized mean difference (SMD), which measures the incremental A1c reduction by the intervention, above and beyond usual care, in standard deviation units. The pooled SMD we report is usually considered to reflect a small difference between treatment and control groups. ${ }^{27}$ However, as pointed out by Durlak, ${ }^{28}$ rigid categorizations of therapeutic efficacy based on SMD values can be misleading. In addition, although methodologically warranted, the assessment of efficacy of in standard deviation units may be difficult to understand for most readers. Examination of individual study results as unadjusted changes in mean A1c reduction may offer an additional perspective, provided that it is interpreted with due caution. In that sense, eight of the nine studies showed a larger unadjusted mean Alc reduction in the $\mathrm{CHW}$ arm than in the control arm - in relative terms, that reduction was 1.6 to 12 times greater in the CHW arm. Only the study by Tang et al. showed a lower relative mean Alc reduction by CHWs. However, as we already discussed, the design of that study probably handicapped the evaluation against CHWs. Their control arm was not limited to "usual care", but had an intervention carried out by Peer Leaders, who were, by design, in more frequent contact with participants than the CHWs. It should be noted that, given its small sample size, that study was not influential in our meta-analysis, and removing it did not substantially modify our findings.

A comparison with the efficacy reported for other interventions in lowering A1c levels may also be informative. Previous meta-analyses have shown similar effect sizes for A1c reduction by other interventions. Polisena et al. estimated an SMD (95\% CI) of 0.21 (0.08 to 0.35$)$ for home telemonitoring interventions. ${ }^{29}$ In a meta-analysis by Harkness et al., psychosocial interventions aimed at improving both the physical and mental health of people with diabetes achieved an SMD of $0.29(0.21$ to 0.37$)$ for A1c reduction. ${ }^{30}$ Interventions that applied the Chronic Care Model to diabetes care resulted in a standardized A1c reduction of $0.19(0.10$, $0.29) .^{31}$

The limitations of the available evidence used for our metaanalysis must be considered. First and foremost, the small number of eligible randomized controlled trials resulted in a wide confidence interval for the pooled estimate, limited our ability to rule out publication bias, ${ }^{25,26}$ and reduced the confidence in the meta-regression results. ${ }^{17}$ Second, there was considerable variability in trial design, including target population, intervention components, participation of other health care professionals, trial length, and baseline A1c values of the study participants. However, it is reassuring to note that the outcomes heterogeneity across studies, as measured by the $\mathrm{I}^{2}$ statistic, was moderate, suggesting that in spite of methodological differences, the efficacy estimates, that is, the observed effects of the CHW intervention, were not excessively heterogeneous. In addition, the studies we meta-analyzed reported substantial attrition, but had, in general, a low risk of bias. Several trials did not report allocation concealment during randomization, but this does not necessarily mean allocation was not concealed. For example, a study by Devereaux et al. reviewed 98 randomized trials, and then contacted investigators to identify under-reporting of concealment. They found that allocation concealment had not been reported in $54(55 \%)$ of the publications, but when contacted directly, investigators reported concealing allocation in $96 \%$ of the trials.

Despite the above noted limitations, our meta-analysis provides useful information for health care organizations, and clinicians who are interested in the implementation of CHW programs for diabetes, and for researchers planning studies in this field. In addition, the completion of currently ongoing trials should add much needed information, and increase our confidence in meta-analytic estimates. ${ }^{32-36}$ The efficacy of CHWs may vary depending on whether they work from within the community, or integrated into multidisciplinary health care teams. The CHWs worked in a team that included other health care professionals, either a nurse practitioner or a diabetes nurse, in only four of the nine long-term studies. Only two of those nine studies assessed the CHW intervention as a "stand alone" protocol, in which the CHW intervention did not actively involve the participant's PCP. ${ }^{12,21}$ At this point, there is insufficient data to determine whether the efficacy of a CHW intervention program is enhanced through the collaboration with other health care professionals. Of note, there is a strong movement, supported by public health experts, third party payers, and governmental institutions, towards the implementation of a patient-centered Medical Home. ${ }^{37}$ It is thus relevant to assess the participation of CHWs in Medical Homes created for disadvantaged populations. Another important question for future research is whether telephone-based interventions may be efficacious when in-person protocols are not feasible.

The great variability that we found in $\mathrm{CHW}$ intervention models in randomized trials is probably a reflection of the heterogeneity seen across CHW clinical programs being 
Table 3. Risk of Bias

\begin{tabular}{|c|c|c|c|c|c|c|}
\hline First Author & $\begin{array}{l}\text { Allocation } \\
\text { Concealment }\end{array}$ & $\begin{array}{l}\text { Balanced Distribution } \\
\text { of Baseline Characteristics }\end{array}$ & $\begin{array}{l}\text { Standardized } \\
\text { A1c } \\
\text { Measurement }\end{array}$ & $\begin{array}{l}\text { Attrition Control } \\
\text { Arm }\end{array}$ & $\begin{array}{l}\text { Attrition } \\
\text { Intervention } \\
\text { Arm }\end{array}$ & $\begin{array}{l}\text { Intention to } \\
\text { Treat Analysis }\end{array}$ \\
\hline Corkery & Not reported & Not reported & Yes & $53 \%$ & $20 \%$ & No \\
\hline Lujan & Not reported & $\begin{array}{l}\text { Higher proportion covered } \\
\text { by insurance in control arm, } \\
\text { addressed in the analysis }\end{array}$ & Yes & $6 \%$ & $5 \%$ & No \\
\hline Babamoto & $\begin{array}{l}\text { No (random } \\
\text { numbers table) }\end{array}$ & $\begin{array}{l}\text { Higher proportion of } \\
\text { females in PCP control } \\
\text { arm }\end{array}$ & Not reported & $\begin{array}{l}50 \% \text { in PCP arm, } \\
43 \% \text { in nurse case } \\
\text { manager arm }\end{array}$ & $28 \%$ & No \\
\hline Spencer & Not reported & $\begin{array}{l}\text { Older age, lower self- } \\
\text { efficacy in control arm; } \\
\text { analysis did not adjust } \\
\text { for self-efficacy }\end{array}$ & No (chart abstraction) & $16 \%$ & $18 \%$ & Yes \\
\hline Brown & Not reported & Yes & Yes & $10 \%$ & $11 \%$ & Yes \\
\hline Gary & Yes & Yes & Yes & $7 \%$ & $13 \%$ & Yes \\
\hline Allen & Not reported & $\begin{array}{l}\text { Higher A1c in intervention } \\
\text { arm, addressed in the analysis }\end{array}$ & Yes & $\approx 6 \%{ }^{*}$ & $\approx 6 \%{ }^{*}$ & Yes \\
\hline Prezio & Yes & Yes & Yes & $13 \%$ & $13 \%$ & Yes \\
\hline DePue & Not reported & $\begin{array}{l}\text { Higher alcohol consumption, } \\
\text { and less doctor visits for } \\
\text { diabetes care in CHW group; } \\
\text { addressed in analysis }\end{array}$ & Yes & $10 \%$ & $9 \%$ & Yes \\
\hline Rothschild & Yes & Yes (for relevant covariates) & Yes & $13 \%$ & $19 \%$ & Yes \\
\hline Perez-Escamilla & Yes & Yes & Yes & $29 \%$ & $22 \%$ & Yes \\
\hline Tang & Yes & Yes & Yes & $30 \%$ & $27 \%$ & Yes \\
\hline Palmas & Yes & Yes & Yes & $12 \%$ & $19 \%$ & Yes \\
\hline
\end{tabular}

*Exact numbers not reported

CHW Community Health Worker; PCP Primary Care Provider

implemented across the United States. ${ }^{38}$ There is widespread consensus regarding the need for an evidence-based approach to standardize the training curriculum and certification of CHWs, and to validate the protocols and tools they implement in their work, while preserving the flexibility to adapt to the specific needs of the communities they serve. System-based initiatives that integrate CHWs into multidisciplinary teams to provide care to underserved populations have already been implemented across the United States, but with great variability in resources. There remains a great need for systematic support and development in most states. A comprehensive review in Massachusetts, published in 2010, identified four areas in which public health officials should act to achieve efficacious and sustainable CHW models: 1) development of appropriate infrastructure, 2) providing CHWs with a professional identity through clear definition of core competencies and roles in the healthcare system, 3) workforce development with training, certification, and continuing education, and 4) financing. ${ }^{6}$ One essential component in the development of sustainable CHW workforce is the guidance and financial support provided by State governments. A 2013 review by the CDC showed that only seven states had laws authorizing

Table 4. Meta-Analysis of Within-Group Mean A1c Reduction by CHW Interventions Over at Least 12 Months

\begin{tabular}{|c|c|c|c|c|}
\hline First Author & $\begin{array}{l}\text { Mean (SD) A1c Reduction } \\
\text { in Intervention Arm } \\
\mathrm{N}\end{array}$ & $\begin{array}{l}\text { Mean (SD) A1c reduction } \\
\text { in Control Arm } \\
\mathrm{N}\end{array}$ & Weight (\%) & $\begin{array}{l}\text { Standardized Mean Difference } \\
(95 \% \text { confidence interval) }\end{array}$ \\
\hline Brown & $\begin{array}{l}0.89(0.26) \\
126\end{array}$ & $\begin{array}{l}0.07(2.95) \\
126\end{array}$ & 9.7 & $0.40(0.15,0.65)$ \\
\hline Gary & $\begin{array}{l}0.20(1.70) \\
273\end{array}$ & $\begin{array}{l}0.08(1.93) \\
269\end{array}$ & 21.3 & $0.07(-0.10,0.23)$ \\
\hline Allen & $\begin{array}{l}0.60(2.30) \\
264\end{array}$ & $\begin{array}{l}0.10(1.80) \\
261\end{array}$ & 20.5 & $0.24(0.07,0.41)$ \\
\hline Prezio & $\begin{array}{l}1.60(2.24) \\
90\end{array}$ & $\begin{array}{l}0.95(2.31) \\
90\end{array}$ & 7.0 & $0.28(-0.01,0.58)$ \\
\hline DePue & $\begin{array}{l}0.31(1.68) \\
95\end{array}$ & $\begin{array}{l}0.03(1.50) \\
148\end{array}$ & 9.1 & $0.17(-0.08,0.44)$ \\
\hline Rothschild & $\begin{array}{l}0.96(2.07) \\
73\end{array}$ & $\begin{array}{l}-0.12(1.66) \\
71\end{array}$ & 5.4 & $0.57(0.24,0.90)$ \\
\hline Perez-Escamilla & $\begin{array}{l}0.86(1.89) \\
105\end{array}$ & $\begin{array}{l}0.34(2.42) \\
106\end{array}$ & 8.2 & $0.24(-0.03,0.51)$ \\
\hline Tang & $\begin{array}{l}0.39(0.89) \\
60\end{array}$ & $\begin{array}{l}0.55(1.60) \\
56\end{array}$ & 4.6 & $-0.12(-0.56,0.31)$ \\
\hline Palmas & $\begin{array}{l}0.29(1.70) \\
179\end{array}$ & $\begin{array}{l}0.07(1.58) \\
181\end{array}$ & 14.1 & $0.13(-0.07,0.34)$ \\
\hline Overall & & & & $\begin{array}{l}0.21(0.11,0.32) \\
\text { Heterogeneity I }^{2}=0.37\end{array}$ \\
\hline
\end{tabular}




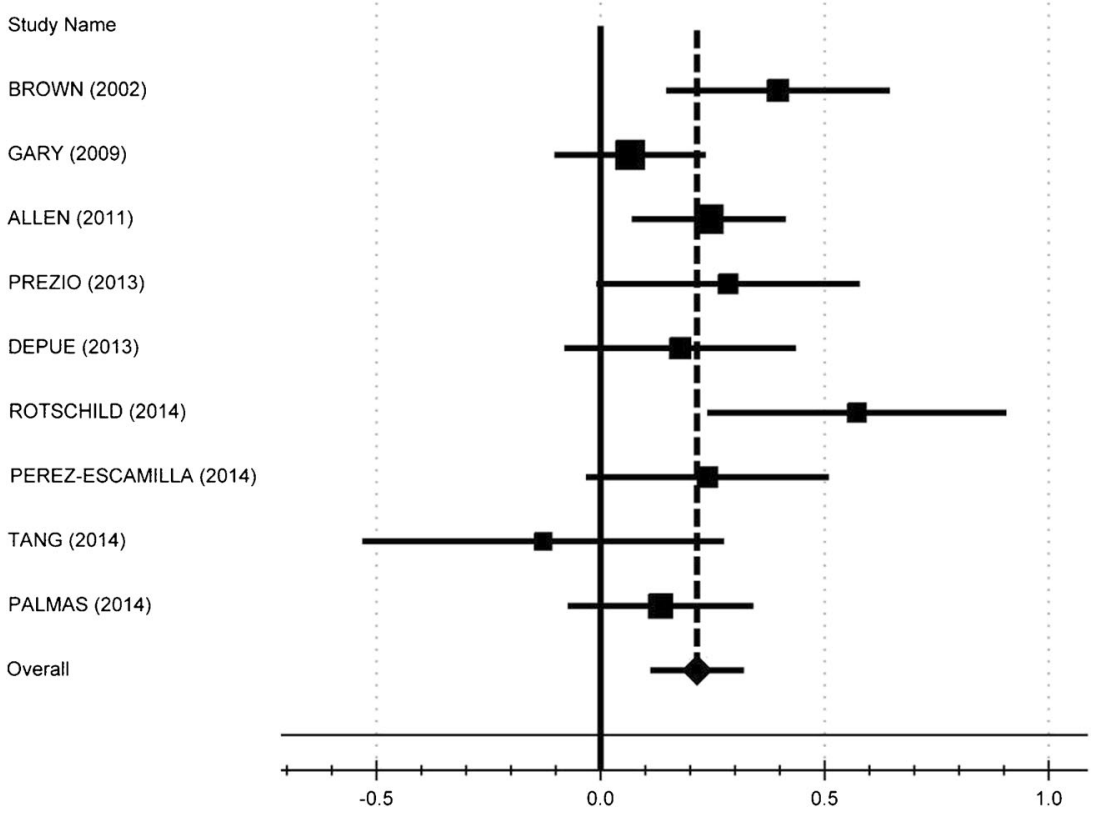

Figure 2 Forest plot for A1c reduction by community health worker interventions in longer-term studies.

Medicaid to reimburse for CHW services. ${ }^{39}$ In addition, only five states had enacted legislation to create a CHW certification process or required $\mathrm{CHWs}$ to be certified. The work carried out in the state of Massachusetts could be used as a model by others, as it addressed to four core areas delineated above. More recently, the Affordable Care Act has provided new opportunities. A review by Katzen and Morgen, from the Center for Health Law and Policy Innovation at Harvard, identified three ways three ways the Affordable Care Act (ACA) has "opened doors for CHWs". ${ }^{40}$ First, the ACA has increased access to preventive health services under Medicaid, and has clarified that states may designate non-licensed providers (i.e., CHWs) to provide preventive services. Second, the ACA offers state Medicaid programs the opportunity to create "Health Homes" for beneficiaries living with chronic illness, and those homes may include a role for CHWs. Third, the ACA created funding for State Innovation Models to help states improve health outcomes and quality of care while slowing growth in health costs; at the time of the report, four states had included CHWs in their innovation models.

In conclusion, our meta-analysis of $\mathrm{CHW}$ interventions has found a modest reduction in $\mathrm{A} 1 \mathrm{c}$, as compared to usual care. The estimated effect was larger in studies with higher mean baseline A1c, suggesting that people with poorer glycemic control may benefit more.

Acknowledgements: These analyses were supported by grants $P 60$ MD000206 and P60 MD000206-08S1 from the National Institute on Minority Health and Health Disparities.

Conflict of Interest: The authors declare that they do not have a conflict of interest.

Author contributions: WP, OC, and JL conceived the meta-analysis. WP had the primary analytical and writing responsibilities. $O C$ and $J L$ edited the manuscript. DM and SD assisted WP with the search of studies for the meta-analysis and performed independent reviews of individual studies. SF assisted with the writing of the manuscript and review of community health worker literature. JT provided statistical supervision.

Corresponding Author: Walter Palmas, MD, MS, FAHA; Department of MedicineColumbia University Medical Center, PH9 East, room 107, 622 West 168th Street, New York, NY 10032, USA (e-mail:wp56@cumc.columbia.edu).

\section{REFERENCES}

1. Harris MI. Racial and ethnic differences in health care access and health outcomes for adults with type 2 diabetes. Diabetes Care. 2001;24:454-9.

2. Mainous AG 3rd, Baker R, Koopman RJ, et al. Impact of the population at risk of diabetes on projections of diabetes burden in the United States: an epidemic on the way. Diabetologia. 2007;50:934-40.

3. Perez LM, Martinez J. Community health workers: social justice and policy advocates for community health and well-being. Am J Public Health. 2008;98:11-4.

4. Lewin SA, Dick J, Pond P, et al. Lay health workers in primary and community health care. Cochrane Database Syst Rev. 2005;CD004015.

5. Norris SL, Chowdhury FM, Van Le K, et al. Effectiveness of community health workers in the care of persons with diabetes. Diabet Med $\mathrm{J} \mathrm{Br}$ Diabet Assoc. 2006;23:544-56.

6. Rosenthal EL, Brownstein JN, Rush CH, et al. Community health workers: part of the solution. Health Aff. 2010;29:1338-42.

7. Whitley EM, Everhart RM, Wright RA. Measuring return on investment of outreach by community health workers. J Health Care Poor Underserved. 2006; 17:6-15.

8. Spencer MS, Rosland AM, Kieffer EC, et al. Effectiveness of a community health worker intervention among African American and Latino adults with type 2 diabetes: a randomized controlled trial. Am J Public Health. 2011;101:2253-60.

9. Lujan J, Ostwald SK, Ortiz M. Promotora diabetes intervention for Mexican Americans. Diabet Educ. 2007;33:660-70.

10. Brown SA, Garcia AA, Kouzekanani K, Hanis CL. Culturally competent diabetes self-management education for Mexican Americans: the Starr County border health initiative. Diabetes Care. 2002;25:259-68.

11. Allen JK, Dennison-Himmelfarb CR, Szanton SL, et al. Community Outreach and Cardiovascular Health $(\mathrm{COACH})$ Trial: a randomized, controlled trial of nurse practitioner/community health worker cardiovascular disease risk reduction in urban community health centers. Circ Cardiovasc Qual Outcomes. 2011;4:595-602. 
12. Rothschild SK, Martin MA, Swider SM, et al. Mexican American Trial of Community Health Workers: A Randomized Controlled Trial of a Community Health Worker Intervention for Mexican Americans With Type 2 Diabetes Mellitus. Am J Public Health. 2013.

13. Gary TL, Batts-Turner M, Yeh HC, et al. The effects of a nurse case manager and a community health worker team on diabetic control, emergency department visits, and hospitalizations among urban African Americans with type 2 diabetes mellitus: a randomized controlled trial. Arch Intern Med. 2009; 169:1788-94.

14. Babamoto KS, Sey KA, Camilleri AJ, Karlan VJ, Catalasan J, Morisky DE. Improving diabetes care and health measures among hispanics using community health workers: results from a randomized controlled trial. Health Educ Behav. 2009;36:113-26.

15. Scottish Intercollegiate Guidelines Network. A guideline developer's handbook. Avaialable at www.sign.ac.uk/pdf/sign50.pdf. Accessed February 6, 2015.

16. Higgins JPT, Thompson SG, Deeks JJ, Altman DG. Measuring inconsistency in meta-analyses. BMJ. 2003;327:557-60.

17. Thompson SG, Higgins JP. How should meta-regression analyses be undertaken and interpreted? Stat Med. 2002;21:1559-73.

18. Centre TNC. The Nordic Cochrane Centre, The Cochrane Collaboration. Review Manager (RevMan). In: The Nordic Cochrane Centre TCC, ed. The Nordic Cochrane Centre, The Cochrane Collaboration. 5.1 ed. Copenhagen; 2011:Computer Program.

19. Corkery E, Palmer C, Foley ME, Schechter CB, Frisher L, Roman SH. Effect of a bicultural community health worker on completion of diabetes education in a Hispanic population. Diabetes Care. 1997;20:254-7.

20. DePue JD, Dunsiger S, Seiden AD, et al. Nurse-community health worker team improves diabetes care in American Samoa: results of a randomized controlled trial. Diabetes Care. 2013;36:1947-53.

21. Palmas W, Findley SE, Mejia M, et al. Results of the northern Manhattan diabetes community outreach project: a randomized trial studying a community health worker intervention to improve diabetes care in Hispanic adults. Diabetes Care. 2014;37:963-9.

22. Perez-Escamilla R, Damio G, Chhabra J, et al. Impact of a Community Health Workers-Led Structured Program on Blood Glucose Control Among Latinos With Type 2 Diabetes: The DIALBEST Trial. Diabetes Care. 2014.

23. Prezio EA, Cheng D, Balasubramanian BA, Shuval $\mathbf{K}$, Kendzor DE, Culica D. Community Diabetes Education (CoDE) for uninsured Mexican Americans: a randomized controlled trial of a culturally tailored diabetes education and management program led by a community health worker. Diabetes Res Clin Pract. 2013;100:19-28.

24. Tang TS, Funnell M, Sinco B, et al. Comparative effectiveness of peer leaders and community health workers in diabetes self-management support: results of a randomized controlled trial. Diabetes Care. 2014;37:1525-34.

25. Sterne JA, Gavaghan D, Egger M. Publication and related bias in metaanalysis: power of statistical tests and prevalence in the literature. J Clin Epidemiol. 2000;53:1119-29.

26. Egger M, Davey Smith G, Schneider M, Minder C. Bias in meta-analysis detected by a simple, graphical test. BMJ. 1997;315:629-34.
27. Fritz CO, Morris PE, Richler JJ. Effect size estimates: current use, calculations, and interpretation. J Exp Psychol Gen. 2012;141:2-18.

28. Durlak JA. How to select, calculate, and interpret effect sizes. J Pediatr Psychol. 2009;34:917-28.

29. Polisena J, Tran K, Cimon K, Hutton B, McGill S, Palmer K. Home telehealth for diabetes management: a systematic review and metaanalysis. Diabet Obes Metab. 2009;11:913-30.

30. Harkness E, Macdonald W, Valderas J, Coventry P, Gask L, Bower P. Identifying psychosocial interventions that improve both physical and mental health in patients with diabtes: a systematic review and metaanalysis. Diabetes Care. 2010;33:926-30.

31. Tsai AC, Morton SC, Mangione CM, Keeler EB. A meta-analysis of interventions to improve care for chronic illnesses. Am J Manag Care. 2005; 11:478-88.

32. Carrasquillo O, Patberg E, Alonzo Y, Li H, Kenya S. Rationale and design of the Miami Healthy Heart Initiative: a randomized controlled study of a community health worker intervention among Latino patients with poorly controlled diabetes. Int J General Med. 2014;7:115-26.

33. Rosal MC, White MJ, Restrepo A, et al. Design and methods for a randomized clinical trial of a diabetes self-management intervention for low-income Latinos: Latinos en Control. BMC Med Res Methodol. 2009;9:81.

34. Nelson $\mathbf{K}$, Drain N, Robinson J, et al. Peer Support for Achieving Independence in Diabetes (Peer-AID): design, methods and baseline characteristics of a randomized controlled trial of community health worker assisted diabetes self-management support. Contemp Clinical Trials. 2014;38:361-9.

35. Islam N, Riley $\mathbf{L}$, Wyatt $\mathbf{L}$, et al. Protocol for the DREAM Project (Diabetes Research, Education, and Action for Minorities): a randomized trial of a community health worker intervention to improve diabetic management and control among Bangladeshi adults in NYC. BMC Public Health. 2014; $14: 177$.

36. Cummings DM, Lutes LD, Littlewood K, Dinatale E, Hambidge B, Schulman K. EMPOWER: a randomized trial using community health workers to deliver a lifestyle intervention program in African American women with Type 2 diabetes: design, rationale, and baseline characteristics. Contemp Clin Trials. 2013;36:147-53.

37. Hoff T, Weller W, DePuccio M. The patient-centered medical home: a review of recent research. Med Care Res Rev MCRR. 2012;69:619-44.

38. Cherrington A, Ayala GX, Amick H, Allison J, Corbie-Smith G, Scarinci I. Implementing the community health worker model within diabetes management: challenges and lessons learned from programs across the United States. Diabet Educ. 2008;34:824-33.

39. Centers for Disease Control. A Summary of State Community Health Worker Laws. Available at http://www.cdc.gov/dhdsp/pubs/docs/chw state_laws.pdf. Accessed February 6, 2015

40. Katzen A, Morgan M. Affordable Care Act Opportunities for Community Health Workers. How Medicaid Preventive Services, Medicaid Health Homes, and State Innovation Models are Including Community Health Workers: Harvard University; 2014. Available at http://www.chlpi.org/wpcontent/uploads/2013/12/ACA-Opportunities-for-CHWsFINAL-8-12.pdf. Accessed February 6, 2015 